Journal of Innovative Optical Health Sciences

Vol. 9, No. 3 (2016) 1602001 (3 pages)

(c) The Author(s)

DOI: $10.1142 /$ S1793545816020016

\title{
Editorial - Introduction to the special issue on super-resolution optical microscopy
}

\author{
Published 13 May 2016
}

Super-resolution optical microscopy, sometimes called optical nanoscopy, refers to a new kind of far-field optical microscopy which allows optical imaging with a resolution higher than the well-known resolution limit (or the Abbe diffraction limit) which stood for more than a century. The reported approaches that break the resolution limit involve either effectively reducing the focus region of the excitation light or stochastic separation and precise localization of single fluorophores. Super-resolution optical microscopy provides unprecedented opportunities for tackling outstanding fundamental questions in life science, medicine, materials, and many others that require non-invasive imaging with molecular level spatial resolution.

In the past decade, a large number of studies have been made in the technology development and applications of super-resolution optical microscopy. Among them, researches from China appeared more than ten years ago, ${ }^{1}$ and grew rapidly in the past several years. This special issue is intended to present some of the recent progresses from China. Original research papers and reviews included here cover a broad range of topics: development of new fluorescent probes and labeling strategies, selection and use of low-light cameras, new data analysis and reconstruction methods, new super-resolution imaging methods, and recent applications of super-resolution imaging in glycoscience.

$\mathrm{Xu}$ and his colleagues ${ }^{2}$ in Beijing, China summarized the photophysical and biochemical properties of the recently developed photomodulatable fluorescent proteins (PMFPs), and discussed the properties of PMFPs that are important for different kinds of super-resolution imaging techniques. They also pointed out the need of brighter green PMFPs with enhanced contrast ratios and photostability for live-cell superresolution techniques, and red/far-red PMFPs with optimal photochemical characteristics for dual-color super-resolution imaging.

The Wang group 3 in Changchun, China focused on the developments and challenges of super-resolution imaging in glycoscience, or more specifically, the distribution and role of carbohydrates in cell membranes. They introduced the imaging principle and the available fluorescent probes for super-resolution imaging, and the strategies for labeling carbohydrates. They also summarized the applications of super-resolution imaging technology in spatial investigation of carbohydrates. This review is surely beneficial for researchers who are interested to use super-resolution imaging technology as a tool to obtain new insights into the study of glycoscience.

To help readers understand the selection and optimal use of low-light cameras in super-resolution localization microscopy, one of the Guest Editors and his colleagues ${ }^{4}$ provided a tutorial on how to fully access the performance of low-light cameras using a well-developed method called Photon Transfer Curve (PTC). They introduced briefly the key parameters for characterizing low-light cameras, then explained how to use the PTC method for accessing the entire performance of a low-light camera, as well as other

This is an Open Access article published by World Scientific Publishing Company. It is distributed under the terms of the Creative Commons Attribution 4.0 (CC-BY) License. Further distribution of this work is permitted, provided the original work is properly cited. 
derivative methods for quantifying the performance of individual pixels inside a camera. They also provided examples on the application of the methods in experimentally quantifying the performance of two representative low-light cameras.

Structured illumination microscopy (SIM) is popular in the super-resolution optical microscopy community due to its great potential to meet the various requirements of live cell imaging, although the resolution enhancement of this technique is only twice that of diffraction-limited optical microscopy. Yang and his colleagues in Fuzhou, China presented two papers in this special issue. In the first paper, ${ }^{5}$ they introduced the principle and development of SIM, along with the combination of this technique with other fluorescence imaging techniques. In the second paper, ${ }^{6}$ they analyzed the theory of typical polarized lights in the field of focal spot imaging, and used simulated images of fluorescent beads to demonstrate the influence of polarized light of different modes on the quality of images of SIM and STED (stimulated emission depletion) microscopy.

The generation of structured illumination and the post-processing of data are critical in SIM; however, the latter has not been publicly released yet. Cao et al. from Beijing, China provided a comprehensive and insightful guide to data post-processing. ${ }^{7}$ They investigated the whole image reconstruction process in SIM, and compared the different methods for estimating the initial phase of structured patterns. They found out that the auto-correlation method achieved the highest performance under a realistic signal-to-noise ratio (SNR).

Fluorescence emission difference (FED) microscopy was recently added to the super-resolution optical microscopy community. Compared to the well-known STED, FED requires low power and simple optical setup, and is applicable to a wide variety of available dyes. Kuang and his colleagues ${ }^{8}$ in Hangzhou, China reported three-dimensional fluorescence emission difference (3D-FED) microscopy using a spatial light modulator (SLM). They provided detailed theoretical analysis and simulation tests to characterize the performance of 3D-FED. They verified experimentally that $3 \mathrm{~d}-\mathrm{FED}$ yielded lateral resolution of $140 \mathrm{~nm}$ and axial resolution of approximate $380 \mathrm{~nm}$.

The last paper in this special issue is by Wang and his co-workers from Shanghai, China. ${ }^{9}$ They reported the theory and experiments on how to obtain an optical tube with super-resolution dark spot, which may further enhance the resolution in STED microscopy. They showed experimentally that a super-resolution optical tube can be generated by tightly focusing a binary phase modulated azimuthally polarized laser beam. When comparing with the focused azimuthally polarizer laser beam without using the binary optics, this new optical tube could increase the resolution of STED microscopy by $25 \%$ under the same intensity, or reach the same resolution with a lower intensity.

Finally, we greatly appreciate the efforts and contributions from all of the authors and the editors. We hope that this special issue could be helpful for readers to understand, at least partially, the researches currently being investigated in the super-resolution optical microscopy community in China. We also hope that this special issue may assist in bringing advances and new insights to the development and maturation of super-resolution optical microscopy.

Guest Editors

Zhen-Li Huang

Britton Chance Center for Biomedical Photonics Wuhan National Laboratory for Optoelectronics (WNLO) Huazhong University of Science and Technology Wuhan 430074, P. R. China

E-mail:leo@mail.hust.edu.cn

Ming-Qiang Zhu Wuhan National Laboratory for Optoelectronics (WNLO) Huazhong University of Science and Technology Wuhan 430074, P. R. China

E-mail: mqzhu@hust.edu.cn 


\section{References}

1. W. Chen, F. Xiao, L. Liu, G. Wang, "Model design and parameter optimization of stimulated emission depletion fluorescence microscopy," Acta Optica Sinica 26, 720-725 (2006).

2. M. Zhang, Z. Fu, P. Xu, "Extending the spatiotemporal resolution of super-resolution microscopies using photomodulatable fluorescent proteins," J. Innov. Opt. Health Sci. 9, 1630009 (2016).

3. J. Chen, T. Tong, H. Wang, "Super-resolution imaging in glycoscience: New developments and challenges," J. Innov. Opt. Health Sci. 9, 1630007 (2016).

4. L. Li, M. Li, Z. Zhang, Z.-L. Huang, "Assessing low-light cameras with photon transfer curve method," J. Innov. Opt. Health Sci. 9, 1630008 (2016).

5. J. Chen, C. Qiu, M. You, X. Chen, H. Yang, S. Xie, "Structured illumination microscopy and its new developments," J. Innov. Opt. Health Sci. 9, 1630010 (2016).

6. C. Qiu, J. Chen, Z. Hou, C. Xu, S. Xie, H. Yang, "Effect of light polarization on pattern illumination superresolution imaging," J. Innov. Opt. Health Sci. 9, 1641001 (2016).

7. Q. Yang, L. Cao, H. Zhang, H. Zhang, G. Jin, "Method of lateral image reconstruction in structured illumination microscopy with super resolution," J. Innov. Opt. Health Sci. 9, 1630002 (2016).

8. G. Zhao, Z. Rong, C. Kuang, C. Zheng, X. Liu, "3D fluorescence emission difference microscopy based on spatial light modulator," J. Innov. Opt. Health Sci. 9, 1641003 (2016).

9. J. Xu, L. Jiang, H. Zhu, L. Liu, J. Hu, H. Wang, S. Zhuang, "Experimental generation and observation of a superresolution optical tube," J. Innov. Opt. Health Sci. 9, 1641002 (2016). 